\title{
Experimental absorbable stent permits airway remodeling
}

\author{
Kuo-Sheng Liu, MD, ${ }^{\text {a,b }}$ Yun-Hen Liu, MD, ${ }^{\mathrm{b}}$ Yi-Jie Peng, MD, ${ }^{\mathrm{c}}$ and Shih-Jung Liu, $\mathrm{PhD}^{\mathrm{a}}$
}

Objective: Despite metallic and silicone stents being effective in treating various airway lesions, many concerns still remain. A bioresorbable stent that scaffolds the airway lumen and dissolves after the remodeling process is completed has advantages over metallic and silicone stents. We designed and fabricated a new mesh-type bioresorbable stent with a backbone of polycaprolactone (PCL), and evaluated its safety and biocompatibility in a rabbit trachea model.

Methods: The PCL stent was fabricated by a laboratory-made microinjection molding machine. In vitro mechanical strength of the PCL stents was tested and compared to that of commercial silicone stents. The bioresorbable stents were surgically implanted into the cervical trachea of New Zealand white rabbits $(\mathrm{n}=6)$. Animals received bronchoscopic examination at 1, 2, 4, 8, and 12 weeks after surgery. Histological examination was completed to evaluate the biocompatibility of the stents.

\begin{abstract}
Results: No animals died during the period of study. Distal stent migration was noted in 1 rabbit. In-stent secretion accumulation was found in 2 rabbits. Histological examination showed intact ciliated epithelium and marked leukocyte infiltration in the submucosa of the stented area at 10 and 28 weeks. Stent degradation was minimal, and the mechanical strength was well preserved at the end of 33 weeks.
\end{abstract}

Conclusions: These preliminary findings showed good safety and biocompatibility of the new PCL stent when used in the airway remodeling. PCL could be a promising bioresorbable material for stent design if prolonged degradation time is required. (J Thorac Cardiovasc Surg 2011;141:463-8)

Airway stents have been used widely for the treatment of tracheobronchial stenosis, which is caused by malignant or benign diseases such as intrinsic or extrinsic malignancies, posttraumatic granulations, and/or tracheomalacia. Stenting in the airway can provide the advantages of counteracting scar contracture, promoting a scaffold for epithelium to cover the reconstructed lumen of the airway, and preventing mechanical disruption secondary to movement of the trachea during breathing and swallowing. Since the introduction of T-tube tracheal stent in $1965,{ }^{1}$ silicone stents have been used in treating various airway diseases. ${ }^{2-5}$ However, due to the relatively greater thickness and tubular geometry of the stents, the mucociliary function of epithelium in the covered area can be disturbed, resulting in impaired clearance of secretions and luminal obstructions. Commonly seen complications of silicone stenting include prosthesis migration, granulation formation, and sputum retention..$^{3-5}$ Metallic stents, on the

\footnotetext{
From the Department of Mechanical Engineering, ${ }^{a}$ Chang Gung University, Kwei-Shan, Tao-Yuan, Taiwan; Department of Thoracic and Cardiovascular Surgery, ${ }^{\mathrm{b}}$ Chang Gung Memorial Hospital, Kwei-Shan, Tao-Yuan, Taiwan; and Department of Ophthalmology, ${ }^{c}$ Buddhist Tzu Chi General Hospital, Taipei Branch, Sindian City, Taipei, Taiwan.

Disclosures: Authors have nothing to disclose with regard to commercial support.

K.S.L. and Y.H.L. contributed equally.

Received for publication April 30, 2010; revisions received July 12, 2010; accepted

for publication Aug 1, 2010; available ahead of print Sept 30, 2010.

Address for reprints: Shih-Jung Liu, PhD, Biomaterials Lab, Mechanical Engineer-

ing, Chang Gung University, 259, Wen-Hwa 1st Road, Kwei-San, Tao-Yuan 333,

Taiwan (E-mail: shihjung@mail.cgu.edu.tw).

$0022-5223 / \$ 36.00$

Copyright (c) 2011 by The American Association for Thoracic Surgery

doi:10.1016/j.jtcvs.2010.08.008
}

other hand, are thinner, more flexible, and easier to insert. Because of their mesh-like design, the mucociliary function of epithelium is well preserved, which leads to a better clearance of secretions. Nevertheless, complications including mucus plugging, stenosing granulation formation, tumor in-growth, stent migration, stent fracture, and fistula between the trachea and the brachiocephalic artery ${ }^{6-9}$ have been reported. Furthermore, metallic stents are not amenable to temporary intraluminal placement, once epithelization and incorporation develop, they are difficult to remove. Airway perforation can be a potential disaster during the removal of metallic stents.

An ideal airway stent should reestablish the airway, cause minimal morbidity and mortality, have limited migration but be easily removed if necessary, maintain luminal patency without causing ischemia or erosion into adjacent structures, cause minimal granulation tissue formation, be easy to place, and be economically affordable. ${ }^{10}$ Stents made of bioresorbable materials have advantages over silicone and metallic stents in that they degrade with time and removal of the stents is not necessary. This advantage is even more pronounced in pediatric patients because as children grow, the existing airway stents may need to be removed or replaced by larger ones. Furthermore, a variety of drugs can also be incorporated into bioresorbable stents for controlled release and targeted local delivery, giving them great potential for various clinical applications.

The purpose of this report is to develop bioresorbable stents for airway remodeling. A balloon-expandable, meshlike stent with a backbone of polycaprolactone (PCL) was 


\section{Abbreviations and Acronyms \\ $\mathrm{PCL}=$ polycaprolactone \\ $\mathrm{PBS}=$ phosphate buffered saline}

fabricated by a laboratory-made microinjection molding machine. The in vitro mechanical property of the bioresorbable stent was characterized, and the safety and biocompatibility of the stent was evaluated in a rabbit trachea model. The final goal of this research is to develop an absorbable stenting system to provide an improved method for airway remodeling.

\section{MATERIALS AND METHODS \\ Materials}

The polymer used for the fabrication of bioresorbable stents was poly- $\epsilon$ caprolactone with a molecular weight of 80,000 (Sigma-Aldrich, St. Louis, Mo). A DuPont model TA-2000 differential scanning calorimeter was used to characterize the thermal properties of the polymer. The measured results suggested that the melting temperature of the polymer was approximately $60.7^{\circ} \mathrm{C}$.

\section{Fabrication of Bioresorbable Stents}

The PCL stent was fabricated by a laboratory-made microinjection molding machine. ${ }^{11} \mathrm{~A}$ mesh-type stent component, which consists of ring-ellipse-ring units as shown in Figure 1, $A$, was first injection molded by the machine. After molding, each stent component was interconnected with another stent component. By interconnecting 7 components, the assembly was rolled into a mesh tube and the final connecting points were welded by hot spot welding. The fabricated stent was then passed over a commercial balloon dilatation catheter (Coronary Stent Driver 3.00 $\mathrm{mm} \times 12 \mathrm{~mm}$; Medtronic, Inc, Galway, Ireland) for expansion. In the process of balloon inflation, the rings slide over the central ellipse to the sides resulting in expansion of the stent. With the release of balloon pressure, a PCL stent with an axial length of $22 \mathrm{~mm}$ and final internal and external diameters of $5 \mathrm{~mm}$ and $6 \mathrm{~mm}$, respectively, was obtained. Figure 1, $B$, shows the manufacture process of the biodegradable stents.

\section{In Vitro Characterization of PCL Stents}

To evaluate the mechanical properties, compression tests of bioabsorbable stents were carried out at room temperature. Area load was applied over the full length of the stents with a $1500 \mathrm{~mm}^{2}$ plate mounted on a LLOYD tensiometer. The cross-head speed was $1 \mathrm{~mm} / \mathrm{min}$. The deformation rate $(D R \%)$ of the stents is defined as:

$D R(\%)=[($ diameter before loading - diameter after loading $)$

$$
/ \text { (diameter before loading })] \times 100
$$

To compare the PCL stents manufactured in this study with the commercial Dumon silicon stents (Novatech, Grasse, France), both PLC and commercial silicon stents were compressed by a 1 Newton force and the deformation rates were recorded.

In vitro weight variation of PCL stents was monitored by immersing the stents in a phosphate buffer, $0.15 \mathrm{~mol} / \mathrm{L}(\mathrm{pH} 7.4)$, at $37^{\circ} \mathrm{C}$. The samples removed from the solutions at different weeks were weighed after being dried inside an oven for 24 hours. The weight retention $(W R(\%))$ of the stents was calculated according to the following equation:

$W R(\%)=\frac{W}{W_{0}} \times 100$

where $\mathrm{W}_{0}$ and $\mathrm{W}$ were the initial weight of the PCL stents and the weight of the stents at different weeks, respectively. Furthermore, the deformation rate of the eluted stents at different times was also measured by the compression test described earlier.

\section{In Vivo Study}

Six New Zealand white rabbits with an average weight of $3.0 \mathrm{~kg}$ were used in this study. During the surgical procedure, the animals were anesthetized with an intramuscular injection of $1 \mathrm{ml} \%$ Rompun (Bayer HealthCare, Animal Health Division, Leverkusen, Germany) and $1 \mathrm{ml}$ Zoletil 50 (Virbac Laboratories, Carros, France). They maintained spontaneous breathing without intubation. The cervical trachea was exposed. A

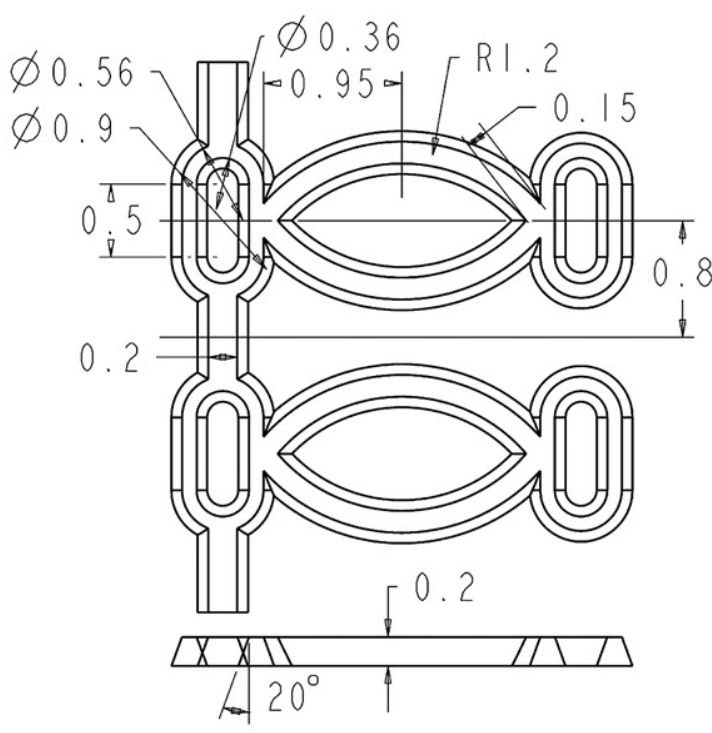

A
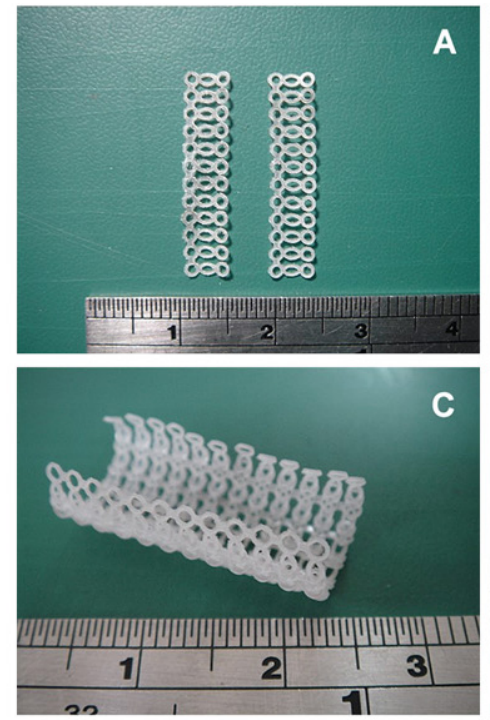

B
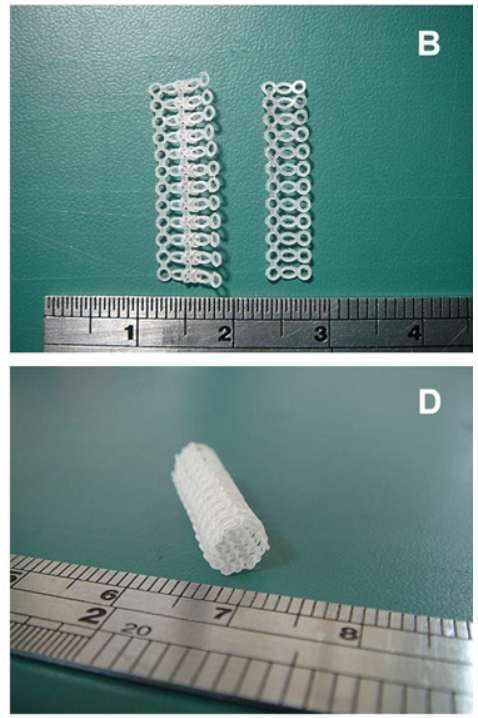

FIGURE 1. A, Dimensions of the stent component. B, Fabrication process of the bioresorbable PCL stents. 
longitudinal incision was made in midline of trachea, crossing six cartilage rings starting from the third. The stent was implanted under direct vision. The tracheotomy was then closed with 7-0 polypropylene suture. All animal procedures received institutional approval and all studied animals were cared for in accordance with regulation of the National Institute of Health of the Republic of China (Taiwan), under the supervision of a licensed veterinarian.

Animals received bronchoscopic examination at 1, 2, 4, 8, and 12 weeks. Three rabbits were killed by intravascular injection of lethal-dose lidocaine at 10 weeks after stent implantation; two were killed at 28 weeks, and the last at 33 weeks. The cervical trachea was excised and submitted for histological examination. Mechanical properties (deformation rates) of the stents retrieved from the animals at 28 and 33 weeks after implant were tested and were compared to those of the in vitro ones.

\section{RESULTS}

\section{In Vitro Evaluation of PCL Stent}

Figure 2 shows the deformation of both PCL stents and commercial silicone stents subjected to a 1 Newton load. The experimental results suggest that the PCL stent exhibited comparable mechanical strength with those of silicone stents.

Figure 3, A, shows the weight retention of PCL stents after immersion in PBS buffer for different times, whereas Figure 3, $B$, shows the mechanical strengths of the eluted stents. No significant mechanical strength and weight loss of the stents was observed after being submerged in PBS for 25 weeks. This might be due to the fact that the degradation period of PCL materials is approximately 2 years. ${ }^{12}$ The degradation and the relevant strength reduction are thus negligible during a 25 -week elution period. Figure 3, A, also shows the compression strengths of PCL stents being implanted in rabbits for 28 and 33 weeks. The measured results suggested that the deformation rate of bioresorbable stents decreased by $35 \%$ and $25 \%$ in vivo at 28 and 33 weeks, respectively, which im-

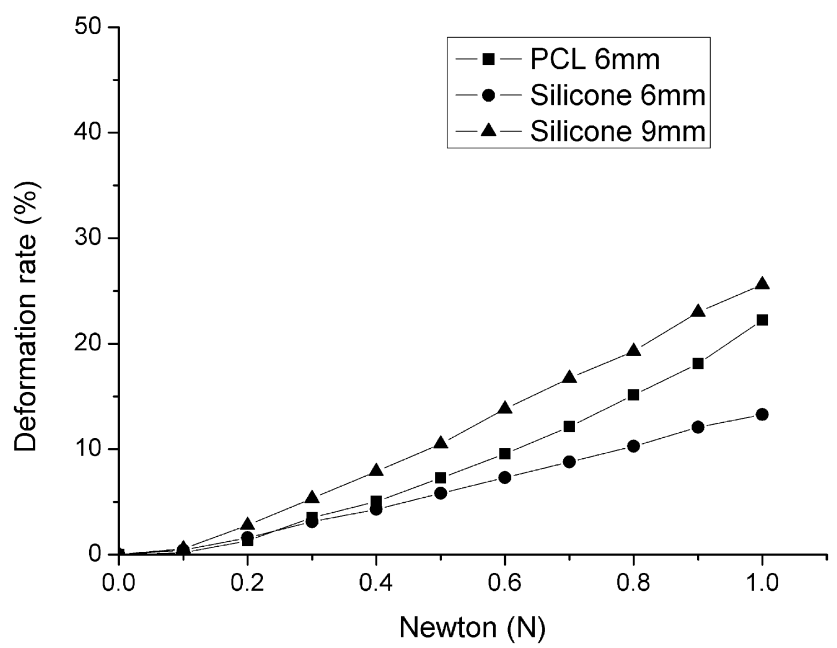

FIGURE 2. A comparison of bioresorbable PCL stents and commercial silicon stents. plies that the PCL materials become stiffer after being implanted in the trachea for some time.

\section{In Vivo Animal Studies}

No animal died of natural causes or airway complications during the period of study. Intermittent stridor was noted at times of agitation in 2 rabbits whose bronchoscopy showed a moderate amount of airway secretion. The activity and appetite of all animals were normal. Obvious weight gain was noted during the period of study.

Figure 4 showed the bronchoscopic findings. Immediately after implantation, there was a small amount of blood in the trachea lumen due to surgical bleeding. Subsequent bronchoscopic pictures revealed excellent lumen patency, with only minimal airway secretions in most of the animals $(n=4)$. We did not observe stent fragmentation in any of the animals.

Distal stent migration was noted in 1 rabbit (Figure 5, $A$ ), which presumably resulted from forceful push by the rigid bronchoscope at the first week. Gradual distal migration was found in serial examinations. At 28 weeks, the distal end of the stent was found at the carina. Moderate amount of airway secretion was noted in 2 rabbits (Figure 5, B), but it was not evident until 8 weeks after operation. In those rabbits with moderate amount of airway secretion, intermittent stridor was audible at times of agitation.

\section{Histological Examination}

At 10 weeks after implantation, marked leukocyte infiltration in the submucosa of the stented area could be seen (Figure 6, A). The ciliary structure of epithelium was maintained. Similar findings were noted at 28 weeks (Figure 6, $B$ ).

\section{DISCUSSION}

Bioresorbable materials have been used in humans as suture and orthopedic or dental implants for several decades. Not until the early 1990s did the clinical application broaden to the field of stents. Until now, the applications of bioresorbable airway stents have been largely experimental. ${ }^{13-18}$ The first literature on a bioresorbable airway stent was reported by Lochbihler ${ }^{13}$ in 1997. The authors designed a bioresorbable stent consisting of polyglycolide (PGA) filaments in a homogenous polydioxanone melt and experimented in a rat trachea model. The stents began to fragment after 4 weeks postimplantation and completely resorbed after 8 weeks. Fourteen percent of the experimental animals died within the first week due to in-stent obstructive granulations.

Korpela ${ }^{14}$ described a new design of spiral stents made of self-reinforced poly-L-lactide (PLLA), and compared with silicone stents in a rabbit model of normal trachea. They found that silicone stents had a tendency to become occluded with encrustation, whereas PLLA stents were welltolerated and caused no foreign body reaction. The PLLA 

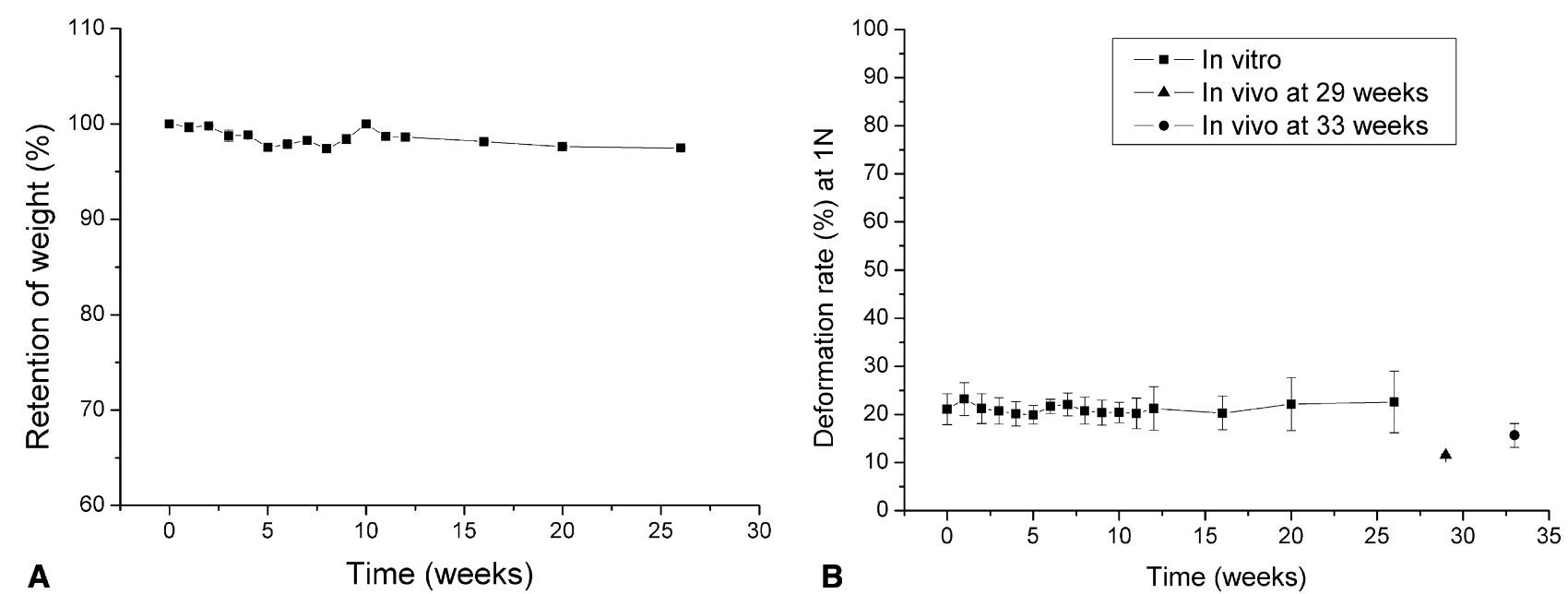

FIGURE 3. A, In vitro weight variation of PCL stents at different times. B, In vitro and in vivo deformation rates of PCL stents at different times.

stents had resorbed by the end of follow-up of 10 months. The authors then tested their stents in a rabbit model of trachea stenosis, which was created by surgical resection of cartilaginous arches of the cervical trachea. ${ }^{15}$ Similar conclusions were made: silicone stents had a tendency to occlude because of internal encrustation and PLLA stents were well-tolerated. Interestingly, one animal in the PLLA stent group developed severe stridor at 26 weeks postimplantation. Autopsy revealed fragments of the spirals of the PLLA stent in the airway lumen. A similar finding was noted in another rabbit that died 31 weeks postsurgery. It was speculated that the PLLA stent fragments might have caused airway obstruction, leading to the animal's death. Robey ${ }^{16}$ described their design of bioresorbable tracheal stent, which was made of 80:20 poly (D,L-lactide-co-glycolide) and was formed in a finger-type shape. In vitro results of their study showed significant mass loss of the stents at 5 weeks, $50 \%$ mass loss at 10 weeks, and complete degradation at 14 weeks. They tested the stents in a rabbit tracheomalacia model, which was created by surgical reconstruction of iatrogenic anterior tracheal wall defect by homologous fascia lata grafts. The stented trachea had a significantly smaller average stenosis $(23 \%)$ across the entire reconstruction site than the control group (34\%) $(P<.05)$. Saito ${ }^{17}$ reported their experiment on bioresorbable PLLA stents. In contrast to previous studies, they designed a tubular knitted stent and tested in a rabbit model of normal trachea. At 40-weeks follow-up, all stents were resorbed. No animals died of airway complications. The new design of tubular knitted stent made possible a lessinvasive, endoscopic implantation. Later, Saito ${ }^{18}$ also successfully deployed their PLLA stent in a dog model using bronchoscope-guided, balloon expansion technique.

Among various bioresorbable materials, PGA, PLLA, PCL, and their copolymers have gained considerable atten- tion as the optimal biomaterials for stents. These polymers differ in their chemical, physical, and biological characteristics. They degrade in biological environments mainly by hydrolysis of the ester linkages, and to a lesser extent by enzymatic digestion. Owing to the high degree of crystallinity and hydrophobicity, the in vivo degradation time of PCL is approximately 2 years. ${ }^{12}$ This characteristic offers a theoretical advantage because the local remodeling process in patients with tracheal stenosis could take more than several months. Biomaterials that degrade too quickly are not suitable for this clinical use.

PCL is nontoxic and tissue-compatible, and can be eventually resorbed in the vital organs. It is also a semicrystalline polymer with a low melting point $\left(59^{\circ} \mathrm{C}\right.$ to $\left.64^{\circ} \mathrm{C}\right)$, and exhibits good flexibility at room temperature and at $37^{\circ} \mathrm{C}$ as compared to other biomaterials such as PLLA and PGA. ${ }^{19}$ This would avoid the occurrence of stent fragmentation ${ }^{15}$ that causes obstruction of the airway. The PLC stents are still fairly flexible despite becoming stiffer in vivo at 33 weeks (Figure 3, B). Furthermore, stents with good flexibility are easier to be manipulated and deployed with minimal invasion, and are more likely to adapt to the bifurcation area.

Histological examination revealed significant inflammation in the stented area, which might contribute to the increased airway secretion in some animals. Theoretically, this problem could be alleviated by incorporation of antiinflammatory drugs in the stent. Furthermore, the meshlike design of PCL stents proposed in this study allowed a bronchoscope-guided placement using the balloon expansion technique. Note that the final step of fabrication of our stent is expansion of the welded stent by an angiodilatation balloon.

We note that in our animal model, the PCL stent was tested with surgical insertion in 6 normal rabbits. Because 

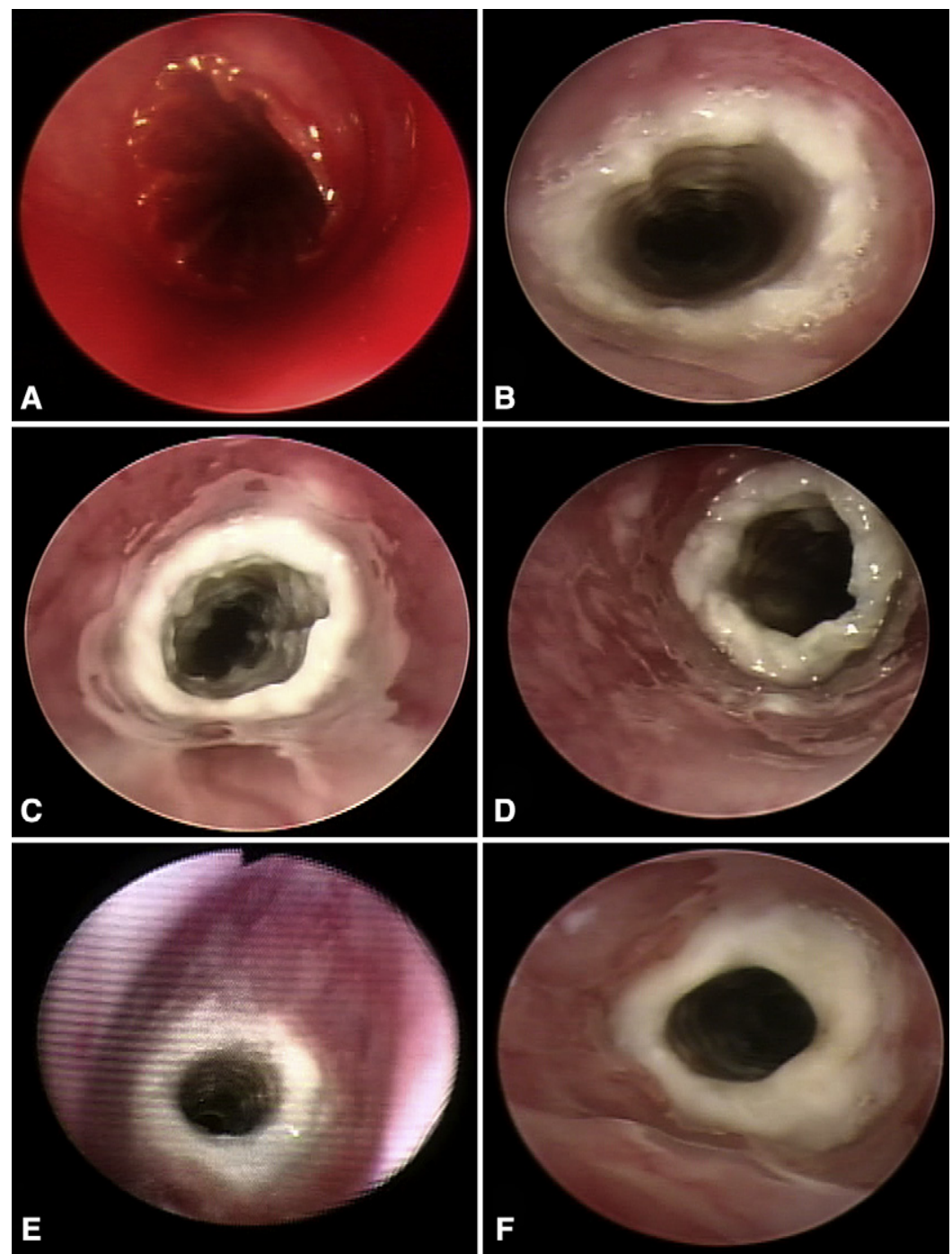

FIGURE 4. A, Immediate postoperative bronchoscopic picture showed blood in the trachea lumen; B, 1 week after surgery; C, 2 weeks after surgery; D, 4 weeks after surgery; E, 8 weeks after surgery; and F, 12 weeks after surgery.

a diseased airway responds differently from a normal windpipe, the stent may not behave the same when the underlying airway is already injured. In addition, only 6 rabbits were tested in our study. More animal tests would be needed to provide a better evaluation of the bioresorbable stents. Further works with a larger number of animals using the minimally invasive balloon-expansion deployment of bioresorbable stents will be the next step of our experiments.
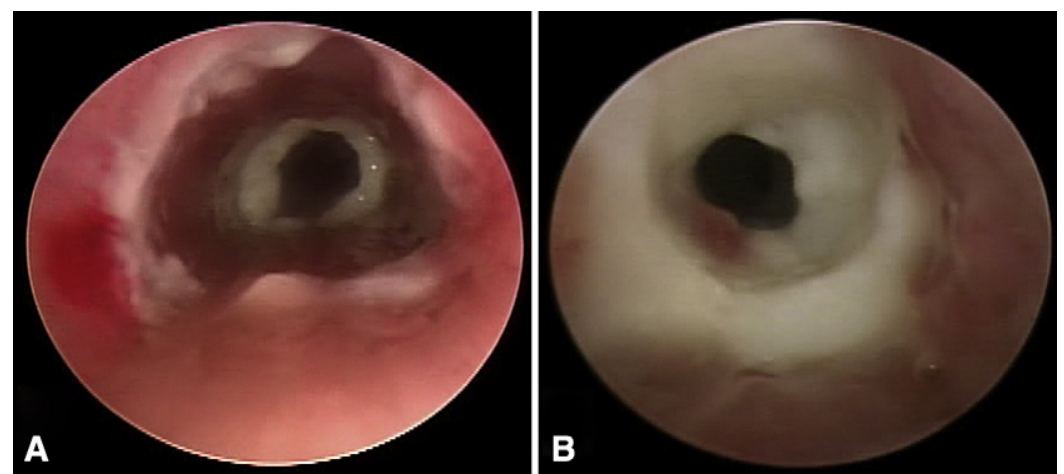

FIGURE 5. A, Distal stent migration due to forceful push by bronchoscope at 1 week after surgery. B, In-stent secretion accumulation at 12 weeks after surgery. 

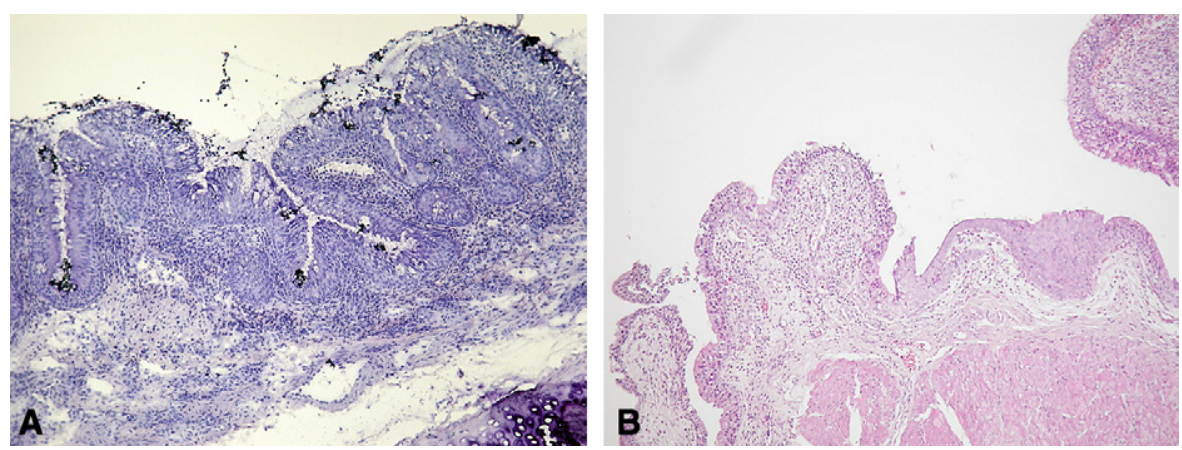

FIGURE 6. A, At 10 weeks after surgery, histological examination showed marked leukocyte infiltration in the submucosa of stented area. B, Submucosa leukocyte infiltration at 28 weeks after surgery.

\section{CONCLUSIONS}

This study has designed and fabricated a new bioresorbable stent with a backbone of PCL, and evaluates its safety and biocompatibility in a rabbit trachea model. A mesh-like PCL stent was fabricated by a lab-made micro injectionmolding machine and then expanded by a balloon to have internal and external diameters of 5 and $6 \mathrm{~mm}$, respectively. The experimental results suggest that the PCL stent exhibited comparable mechanical strength with those of commercial silicone stents. Furthermore, the in vivo animal study showed good safety and biocompatibility of the new PCL stent when used in the airway remodeling. PCL could be a promising bioresorbable material for stent design if the desired degradation time is longer than 1 year.

\section{References}

1. Montgomery WW. T-tube tracheal stent. Arch Otolaryngol. 1965;82:320-1.

2. Dumon JF. A dedicated tracheobronchial stent. Chest. 1990;97:332-48.

3. Martinez-Ballarin JI, Diaz-Jimenez JP, Castro MJ, Moya JA. Silicone stents in the management of benign tracheobronchial stenoses. Tolerance and early results in 63 patients. Chest. 1996;109:626-9.

4. Carretta A, Casiraghi M, Melloni G, Bandiera A, Ciriaco P, Ferla L, et al. Montgomery T-tube placement in the treatment of benign tracheal lesions. Eur J Cardiothorac Surg. 2009;36:352-6.

5. Puma F, Ragusa M, Avenia N, Urbani M, Droghetti A, Daddi N, et al. The role of silicone stents in the treatment of cicatricial tracheal stenoses. J Thorac Cardiovasc Surg. 2000;120:1064-9.

6. Lemaire A, Burfeind WR, Toloza E, Balderson S, Petersen RP, Harpole DH Jr, et al. Outcomes of tracheobronchial stents in patients with malignant airway disease. Ann Thorac Surg. 2005;80:434-8.

7. Breitenbücher A, Chhajed PN, Brutsche MH, Mordasini C, Schilter D, Tamm M. Long-term follow-up and survival after Ultraflex stent insertion in the management of complex malignant airway stenoses. Respiration. 2008;75:443-9.

8. Chung FT, Lin SM, Chen HC, Chou CL, Yu CT, Liu CY, et al. Factors leading to tracheobronchial self-expandable metallic stent fracture. J Thorac Cardiovasc Surg. 2008;136:1328-35.

9. Sihvo EIT, Sioris T, Tynninen O, Salo JA. Fatal fistula between the trachea and the brachiocephalic artery: late complication of a second-generation, self-expanding metallic tracheal stent. J Thorac Cardiovasc Surg. 2006;131: 1415-6.

10. Chin CS, Litle V, Yun J, Weiser T, Swanson SJ. Airway stents. Ann Thorac Surg. 2008;85:S792-6.

11. Liu SJ, Chiang FJ, Hsiao CY, Kau YC, Liu KS. Fabrication of balloonexpandable self-lock drug-eluting polycaprolactone stents using microinjection molding and spray coating techniques. Ann Biomed Eng. 2010;38: 3185-94.

12. Sun H, Mei L, Song C, Cui X, Wang P. The in vivo degradation, absorption and excretion of PCL-based implant. Biomaterials. 2006;27:1735-40.

13. Lochbihler H, Hoelzl J, Dietz HG. Tissue compatibility and biodegradation of new absorbable stents for tracheal stabilization: an experimental study. J Pediatr Surg. 1997;32:717-20.

14. Korpela A, Aarnio P, Sariola H, Tormala P, Harjula A. Comparison of tissue reactions in the tracheal mucosa surrounding a bioabsorbable and silicone airway stents. Ann Thorac Surg. 1998;66:1772-6.

15. Korpela A, Aarnio P, Sariola H, Tormala P, Harjula A. Bioabsorbable selfreinforced poly-L-lactide, metallic, and silicone stents in the management of experimental tracheal stenosis. Chest. 1999;115:490-5.

16. Robey TC, Valimaa T, Murphy HS, Tormala P, Mooney DJ, Weatherly RA. Use of internal bioabsorbable PLGA "finger-type" stents in a rabbit tracheal reconstruction model. Arch Otolaryngol Head Neck Surg. 2000;126: 985-91.

17. Saito Y, Minami K, Kobayashi M, Nakao Y, Omiya H, Imamura H, et al. New tubular bioabsorbable knitted airway stent: biocompatibility and mechanical strength. J Thorac Cardiovasc Surg. 2002;123:161-7.

18. Saito Y, Minami K, Kaneda H, Okada T, Maniwa T, Araki Y, et al. New tubular bioabsorbable knitted airway stent: feasibility assessment for delivery and deployment in a dog model. Ann Thorac Surg. 2004;78:1438-40.

19. Yang S, Leong K-F, Du Z, Chua C-K. The design of scaffolds for use in tissue engineering. Part I. Traditional factors. Tissue Eng. 2001;7:679-89. 